\title{
Transcranial direct current stimulation reduces negative affect but not cigarette craving in overnight abstinent smokers
}

\author{
Jiansong $X u^{1}{ }^{*}$, Felipe Fregni ${ }^{2}$, Arthur L. Brody ${ }^{3}$ and Ardeshir S. Rahman ${ }^{3}$ \\ ${ }^{1}$ Department of Psychiatry, Yale Medical School, New Haven, CT, USA \\ 2 Laboratory of Neuromodulation, Department of Physical Medicine \& Rehabilitation, Spaulding Rehabilitation Hospital \& Massachusetts General Hospital, Boston, \\ MA, USA \\ ${ }^{3}$ Department of Psychiatry \& Biobehavioral Sciences, UCLA School of Medicine, Los Angeles, CA, USA
}

\section{Edited by: \\ Filippo Passetti, Cambridge \\ University, UK}

\section{Reviewed by:}

Przemyslaw Bienkowski, Institute of Psychiatry and Neurology, Poland Andrea Cippitelli, Torrey Pines Institute for Molecular Studies, USA

*Correspondence:

Jiansong Xu, Department of Psychiatry, Yale Medical School, 1 Church Street, Room 729, New Haven, CT 06519, USA

e-mail: jiansong.xu@yale.edu
Transcranial direct current stimulation (tDCS) can enhance cognitive control functions including attention and top-down regulation over negative affect and substance craving in both healthy and clinical populations, including early abstinent $(\sim 1.5 \mathrm{~h})$ smokers. The aim of this study was to assess whether tDCS modulates negative affect, cigarette craving, and attention of overnight abstinent tobacco dependent smokers. In this study, 24 smokers received a real and a sham session of tDCS after overnight abstinence from smoking on two different days. We applied anode to the left dorsolateral prefrontal cortex and cathode to the right supra-orbital area for $20 \mathrm{~min}$ with a current of $2.0 \mathrm{~mA}$. We used self-report questionnaires Profile of Mood States (POMS) to assess negative affect and Urge to Smoke (UTS) Scale to assess craving for cigarette smoking, and a computerized visual target identification task to assess attention immediately before and after each tDCS. Smokers reported significantly greater reductions in POMS scores of total mood disturbance and scores of tension-anxiety, depression-dejection, and confusion-bewilderment subscales after real relative to sham tDCS. Furthermore, this reduction in negative affect positively correlated with the level of nicotine dependence as assessed by Fagerström scale. However, reductions in cigarette craving after real vs. sham tDCS did not differ, nor were there differences in reaction time or hit rate change on the visual task. Smokers did not report significant side effects of tDCS. This study demonstrates the safety of tDCS and its promising effect in ameliorating negative affect in overnight abstinent smokers. Its efficacy in treating tobacco dependence deserves further investigation.

Keywords: tobacco dependence, transcranial direct current stimulation, smoking abstinence, cigarette craving, negative affect, brain stimulation

\section{INTRODUCTION}

Cigarette smoking is the number one cause of preventable death in the United States (1). Most tobacco dependent smokers want to stop smoking (2). However, they often experience tobacco withdrawal symptoms including craving for smoking, negative affect, and deficits in attention after abstinence from smoking, and thus relapses are common after short durations of abstinence (3-5). Current therapies for tobacco dependence include nicotine replacement therapy, bupropion sustained release formula, and varenicline. These therapies can significantly reduce tobacco withdrawal symptoms and increase abstinence rates, but are not effective in all smokers (6-9). Therefore, new therapies for reducing tobacco withdrawal are needed to help smokers quit. This study assessed whether transcranial direct current stimulation (tDCS) could modulate mood, attention, and craving for smoking of tobacco dependent smokers who maintain abstinence overnight.

Transcranial direct current stimulation is a safe, non-invasive, and inexpensive method for modulating neuronal excitability. It modulates cortical excitability using small direct electrical currents $(1 \sim 2 \mathrm{~mA})$ delivered to the scalp (10-14) via two electrodes with opposite polarities (i.e., anodal and cathodal) placed on the scalp. Anodal stimulation increases cortical excitability whereas cathodal stimulation decreases cortical excitability $(12,13,15,16)$. These effects can last up to $90 \mathrm{~min}$ after a single stimulation session of $13 \sim 20 \mathrm{~min}$ (17-20), and can further increase after repeated stimulation (i.e., cumulative effects) $(21,22)$.

Several studies assessed the effects of tDCS on cue-induced craving for drug use. One study applied anodal stimulation of $2 \mathrm{~mA}$ to either the right or left dorsolateral prefrontal cortex (DLPFC) for $20 \mathrm{~min}$. This anodal stimulation reduced cueinduced tobacco craving in early abstinent $(\sim 1.5 \mathrm{~h})$ smokers $(23)$. In another study, daily sessions (20 min) of anodal stimulation to the left DLPFC for 5 days reduced cue-induced tobacco craving and the number of smoked cigarettes during the 5-day period (22). This study also observed the cumulative effect of tDCS, i.e., cue-induced craving decreased further after each tDCS session. Other studies reported that anodal stimulation of the DLPFC 
reduced substance craving of alcohol abusers, marijuana users, and healthy participants (22-28). Furthermore, tDCS has been reported to induce beneficial effects on other cognitive domains such as attention, working memory, or response inhibition in healthy participants or patients with stroke, depression, Parkinson's disease, or alcohol dependence (29-40), and also in affective domains such as mood in patients with depression or tinnitus (36, 41-45).

Findings from aforementioned studies indicate that anodal stimulation of the DLPFC may ameliorate tobacco withdrawal by reducing cigarette craving, ameliorating negative affect, and improving cognitive function. However, only two tDCS studies on smokers have been published, and these two studies assessed smokers abstinent from smoking for a short duration (i.e., $\sim 1.5 \mathrm{~h}$ ). To our knowledge, no published studies assessed the effect of tDCS on longer abstinent smokers (e.g., overnight abstinent) and across several measures related to different aspects of tobacco withdrawal. Given the potential clinical significance of assessing longer abstinent smokers and also to collect pilot data for further larger clinical trials, we designed this study to test whether anodal stimulation applied to the left DLPFC in overnight abstinent smokers would modulate their mood, attention, and craving for smoking. Based on the above-reviewed studies, we predicted that abstinent smokers would show reduced craving and negative mood, and improved performance on an attention task, after real relative to sham tDCS.

\section{MATERIALS AND METHODS \\ PARTICIPANTS}

This study was approved by the Human Investigation Committee at Yale School of Medicine and was performed in accordance with the Declaration of Helsinki. Potential participants were recruited from communities around Yale University through flyers and ads placed on Craigslist (www.craigslist.org). All participants provided written informed consent. The inclusion criteria were good general health, age between 18 and 60 years, more than 10 cigarettes per day for at least 2 years, no current illicit drug use as indicated by negative results on urine drug screens of cocaine, methamphetamine, opiates, or benzodiazepines at all sessions, and $\leq 10$ standard drinks of alcohol per week (one standard drink consists of one $12 \mathrm{oz}$. beer, $6 \mathrm{oz}$. of wine, or one shot $(1.5 \mathrm{oz}$.) of hard liquor ( 80 proof). The exclusion criteria were any current medical conditions, current neuropsychiatric disorders, more than one marijuana cigarette per week, pregnancy as indicated by a positive result on the urine pregnancy test, self-report of learning disability or dyslexia, current use of psychotropic drugs, or self-report of TB or HIV positive.

During a baseline session, carbon monoxide (CO) in expired air was taken as an objective measure of recent smoking (Micro Smokerlyzer II, Bedfont Scientific Instruments), and a level $>15$ ppm was considered as consistent with recent smoking. Participants also completed the Fagerström Test for Nicotine Dependence (46), Urge to Smoke (UTS) Scale (47), Profile of Mood States (POMS) (48), questionnaires for smoking history and demographic information, and received training in performing a computer task testing attention. The final sample included 24 smokers ( 3 females), with a mean age of 45 years (range $28 \sim 59$, standard deviation, $\mathrm{SD}=7.6$ ), smoked an average of 16.4 cigarettes per day (range
$10 \sim 30, \mathrm{SD}=5.6$ ), and a mean Fagerström score of 5.7 (range $1 \sim 9, \mathrm{SD}=2.0$ ).

\section{PROCEDURE}

Subsequent to baseline assessments, subjects participated in two test sessions, one for real and the other for sham tDCS, on two different days with a minimal interval of $48 \mathrm{~h}$. This used a single-blind design, i.e., participants were blind to real vs. sham tDCS. The sequence of the two tDCS sessions was counterbalanced among participants. On each test day smokers reported to the laboratory in the morning around 10 a.m. after maintaining abstinence from smoking overnight ( $>10 \mathrm{~h}$ abstinence). They provided a breath sample for CO assay, which should be $<10$ ppm or half of the baseline measure as a confirmation of overnight abstinence. Otherwise, the study session would be stopped and rescheduled. Smokers also provided a urine sample for drug screen, and would be excluded from further study if the urine sample were positive for any drugs mentioned above.

After confirmation of overnight abstinence and no drug use, participants continued the study session by completing a set of questionnaires including UTS (47) and POMS (48). Then, they performed a computerized task testing attention. After the task, they watched cigarette smoking-related pictures and video clips to induce craving for smoking for $5 \mathrm{~min}$. While viewing smoking cues, smokers were instructed to put a pack of cigarettes on the desk in front of them, hold a cigarette, and put the cigarette in their mouth, and light a lighter without lighting the cigarette. Participants received tDCS (either real or sham) after watching the smoking cues. Following the tDCS, they completed the tDCS Adverse Effects Questionnaire, performed the computerized attention task, and completed the UTS and POMS again.

\section{TRANSCRANIAL DIRECT CURRENT STIMULATION}

We used a $1 \times 1$ Low-Intensity DC Stimulator, Model 1224-B (Soterix, LLC, New York, NY, USA), and two sponge electrodes $(5 \mathrm{~cm} \times 7 \mathrm{~cm})$ soaked with saline to deliver tDCS. During each session, the anode was placed over the left DLPFC and the cathode was placed over the contralateral supra-orbital area. The DLPFC was localized using the international 10/20 EEG system (F3) (49). In real tDCS session, stimulation was given at $2 \mathrm{~mA}$ for $20 \mathrm{~min}$, with gradual ramping up of the current over $30 \mathrm{~s}$. For sham stimulation, current ramped up to $2 \mathrm{~mA}$ over the first $30 \mathrm{~s}$ and then ramped down to zero during another $30 \mathrm{~s}$, thus giving the same initial sensation of tDCS. This procedure was regularly used to keep participants blind to the real vs. sham stimulation $(23,50-52)$. The sham stimulation also lasted for $20 \mathrm{~min}$.

\section{MEASURES}

The POMS consists of six subscales, collectively including 65 fivepoint items that describe mood, and these items were used to calculate a score for total mood disturbance. The range of possible scores is -32 to 200 for total mood disturbance, calculated by subtracting the score for the vigor-activity subscale from the sum of scores for the remaining five subscales (5). The possible scores for the six subscales are as follows: tension-anxiety (0-36), depression-dejection (0-60), anger-hostility (0-48), vigor-activity (0-32), fatigueinertia (0-28), and confusion-bewilderment (0-28). For the total 
mood disturbance and all subscales, except for vigor-activity, a higher score indicates a more negative mood state.

The UTS was used to assess cigarette craving. It consists of following seven-point items: (1) If you could smoke freely, would you like a cigarette at this moment? (2) Do you have an urge for a cigarette right now? (3) Do you miss a cigarette? (4) I crave a cigarette right now, (5) I am going to smoke as soon as possible, (6) All I want right now is a cigarette, (7) I don't want to smoke now, (8) I have no desire for a cigarette now, (9) Nothing would be better than smoking a cigarette right now, (10) Smoking a cigarette would not be pleasant. The highest possible score is 70, and a higher score indicates greater craving. The tDCS Side Effect Questionnaire was used to assess tDCS side effects including headache, neck pain, scalp pain, scalp burns, tingling, skin redness, sleepiness, trouble concentrating, acute mood change, and other effects (24). The severity of each side effect is indexed using a four-point system, i.e., 1-Absent, 2-Mild, 3-Moderate, and 4-Severe.

The computerized task for testing attention had two load conditions, one for low and the other for high load. It used digits, inclusively between 1 and 9, as stimuli. The low load condition presented one digit in the center of the screen for each stimulus (Figure 1), and the stimulus was a target if the digit was an even number. The high load condition presented five digits simultaneously for each stimulus (Figure 1), and the stimulus was a target if three of the five digits were even numbers. The task used block design and each block consisted of 40 trials and one third of them were targets. Each stimulus was presented for $500 \mathrm{~ms}$ with an interstimulus interval of $1000 \mathrm{~ms}$. Our previous studies and others' indicated that different neural substrates underlay attention at different levels of attentional demand $(53,54)$. Therefore, a task with parametric loads was used to help understand whether tDCS modulates attention at specific task loads. Due to a technical problem, task performance record was not complete for four participants. Given that this missing data can be considered completely at random, the task performances of these 4 participants were excluded from analysis, and the performance data from 20 remaining participants were analyzed.

\section{DATA ANALYSIS}

SPSS paired $t$-test was used to compare scores on POMS and UTS between baseline and after overnight abstinence before tDCS. SPSS general Linear Model (GLM) for repeated measures was used to analyze the scores for POMS and UTS, and reaction time (RT) and hit rates on the attention task for assessing the effect of anodal stimulation of the left DLPFC. Because the current study assessed

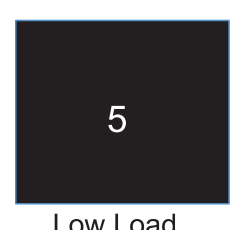

Low Load

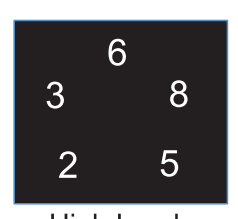

High Load
FIGURE 1 | Task stimulus. One and five digits were presented on the screen as stimulus for the low and high load conditions, respectively. tobacco withdrawal symptoms of each participant repeatedly for four times in total, i.e., two times for each of two study sessions. Therefore, the difference among group means of four assessments can be tested using one-way analysis of variance (ANOVA) with repeated measures, which is implemented using GLM for repeated measures in SPSS. The scores one POMS and UTS and performance parameters were dependent variables, and test sessions and blocks (i.e., pre- vs. post-tDCS) were within-subject variables. Statistical analyses were performed to assess whether dependent variables showed significant differences in changes from before to after stimulation in real vs. sham tDCS session. SPSS binary correlation analysis was used to assess the relationship between scores on Fagerström test and changes in scores of POMS and UTS during each tDCS session. The difference in coefficients of correlations between Fagerström scores and reduction of POMS total scores after real vs. sham stimulation was assessed using a tool from a public website (55). The rates of tDCS side effects between the real and sham sessions were compared using SPSS Chi-square test. All statistical significant thresholds were set at $p<0.05$.

\section{RESULTS}

\section{tDCS EFFECTS}

Relative to baseline, participants did not report significantly greater scores on POMS after overnight abstinence before tDCS on both days of real and sham stimulation (Table 1). Several POMS scores showed a main effect of block, i.e., a significant reduction after tDCS relative to before tDCS. They included the total score $[F(1,23)=13.41, p=0.001]$ (Figure 2B) and scores for subscales tension-anxiety $[F(1,23)=23.36, p<0.001]$ and anger-hostility $[F(1,23)=10.25, p=0.004]$ (Figures 2C,E). Furthermore, several POMS scores showed a significant effect of session $\times$ block twoway interaction, i.e., a greater reduction after real relative to sham tDCS. They included POMS total scale $[F(1,23)=7.7, p=0.011]$ and subscales for tension-anxiety $[F(1,23)=5.1, p=0.033]$, depression-dejection $[F(1,23)=9.2, p=0.006]$, and confusionbewilderment $[F(1,23)=4.9, p=0.037]$ (Figures 2B,C,F,G). The Fagerström score of smokers positively correlated with the reduction of POMS total scores after real stimulation $(N=24$, $r=0.451, p=0.027)$, but not after sham stimulation $(N=24$, $r=-0.285, p=0.176$ ) (Figure 3). The correlations between Fagerström scores and reduction of POMS total scores after real vs. sham stimulation tend to be different significantly $(Z=1.76, p=0.078)$. Abstinent smokers did not report significant tDCS-related changes in scores for subscales fatigueinertia $[F(1,23)=0.74, p=0.398]$ and vigor-activity $[F(1$, 23) $=1.03, p=0.32]$.

Relative to baseline, participants reported a significantly greater score on UTS after overnight abstinence before tDCS on both days of real and sham stimulation (Table 1). Score on UTS showed a main effect of block $[F(1,23)=13.8, p=0.001]$, i.e., a significant reduction after tDCS relative to before tDCS (Figure 2A). However, it did not show an interaction effect of session $\times$ block $[F(1,23)=0.009, p=0.927]$ (Figure 2). The changes of UTS score after real vs. sham tDCS did not correlate with each other significantly $(N=24, r=0.066, p=0.76)$. Participants did not show significant correlations between Fagerström scores and changes on UTS after real $(N=24, r=0.23, p=0.279)$ or sham $(N=24$, 
Table 1 | UTS and POMS scores at baseline and before tDCS at each session.

\begin{tabular}{|c|c|c|c|c|c|c|c|c|c|}
\hline & & UTS & POMS total & $\mathbf{T}-\mathbf{A}$ & A-H & F-I & D-D & C-B & V-A \\
\hline Baseline & & $44.4(16.2)$ & 3.3 (18.8) & $5.0(4.4)$ & $2.6(3.2)$ & $3.9(4.0)$ & $4.7(5.4)$ & $4.1(3.0)$ & $17.0(5.7)$ \\
\hline Real & & $57.4(11.5)$ & $7.8(25.2)$ & $7.6(6.4)$ & $3.2(4.4)$ & $4.4(5.3)$ & $4.0(5.5)$ & $4.3(3.8)$ & $15.6(7.1)$ \\
\hline \multirow[t]{2}{*}{ Baseline vs. real } & $t$ & 4.7 & 0.94 & 1.60 & 0.73 & 0.79 & 0.63 & 0.22 & 1.12 \\
\hline & $p$ & $<0.001$ & 0.36 & 0.12 & 0.47 & 0.44 & 0.54 & 0.83 & 0.27 \\
\hline
\end{tabular}

Number in the parenthesis indicates standard deviation (SD). Abbreviations: $A-H$, anger-hostility; C-B, confusion-bewilderment; $D-D$, depression-dejection; F-I, fatigue-inertia; T-A, tension-anxiety; $V-A$, vigor-activity.

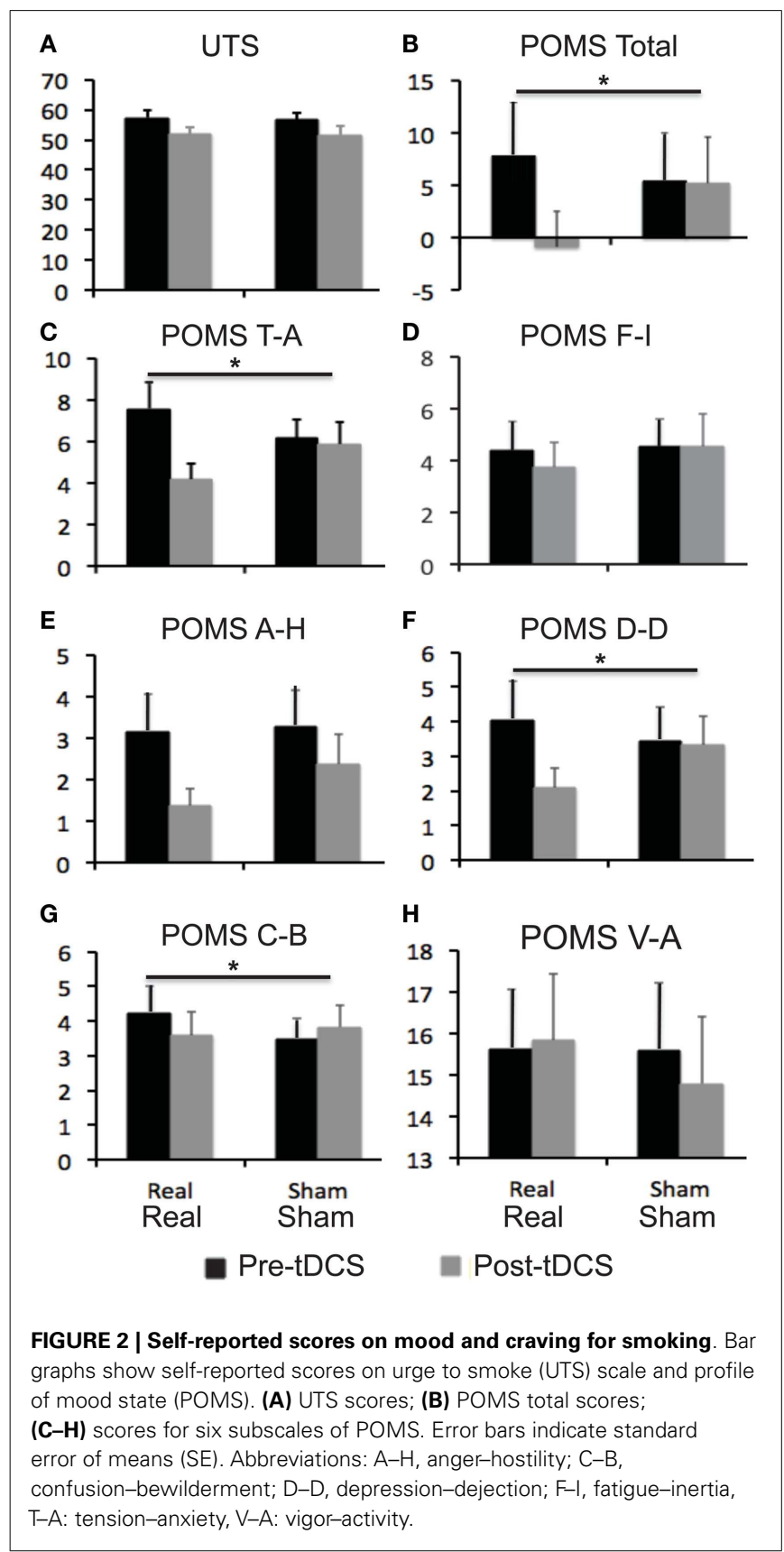

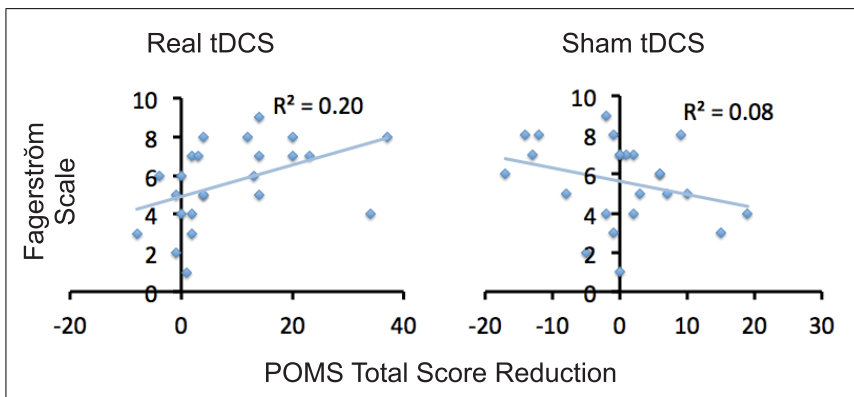

FIGURE 3 | Correlations between level of nicotine dependence and tDCS-induced reduction of negative affect. Scatter plots demonstrate correlations between scores on Fagerström and reduction in POMS total scores after tDCS relative to before tDCS.

$r=-0.152, p=0.479)$ stimulation, nor between changes on scores of UTS and POMS after real $(N=24, r=0.251, p=0.237)$ or sham $(N=24, r=0.330, p=0.115)$ stimulation.

Participants did not show a significantly different change in any performance measures of the attention task at either task load after real relative to sham stimulation (Figure 4).

\section{tDCS SIDE EFFECTS}

The most commonly reported side effects were tingling, sleepiness, and scalp burn (Table 2). These side effects were usually mild and did not prevent any participants from completing tDCS (Table 2). Though more participants reported tingling and sleepiness in the real relative to sham session, this difference between the two sessions did not reach statistical significance, indicating that the blinding method was effective for the current participants.

\section{DISCUSSION}

To our knowledge, this study is the first to assess the effect of tDCS on mood, attention, and craving for smoking of tobacco dependent smokers after overnight abstinence. The main finding was that anodal stimulation to the left DLPFC reduced negative affect of overnight abstinent smokers. This reduction in negative affect positively correlated with the level of nicotine dependence as measured by the Fagerström test. However, tDCS did not show significant effect on cigarette craving or performance on a visual attentional task. 


\section{NEGATIVE AFFECT}

Chronic smoking may impair structure and function of the brain including the DLPFC. For example, chronic smoking desensitizes nicotinic acetylcholinergic receptors and increases their density in the brain including the DLPFC (56-59), reduce gray matter density in the DLPFC $(60,61)$, and alter task-related activity of the DLPFC during an attentional task (62). Overnight abstinence from smoking may further alter functional activity of the DLPFC and other brain regions in tobacco dependent smokers and leads to withdrawal symptoms (63-67). The smokers in the current study reported an increased total score of POMS after overnight abstinence relative to baseline, though this increase did not reach the threshold of statistical significance. The short duration of abstinence $(\sim 10 \mathrm{~h})$ of current study may contribute to this nonsignificant increase in negative affect, because tobacco dependent smokers often reported a significant increase in negative affect a longer duration $(\sim 24 \mathrm{~h})$ of abstinence $(68,69)$.

The functional activity in the left and right DLPFC is associated with positive and negative affect, respectively (70-72). Clinical depression is associated with reduced activity in the left DLPFC and increased activity in the right DLPFC (73). Anodal

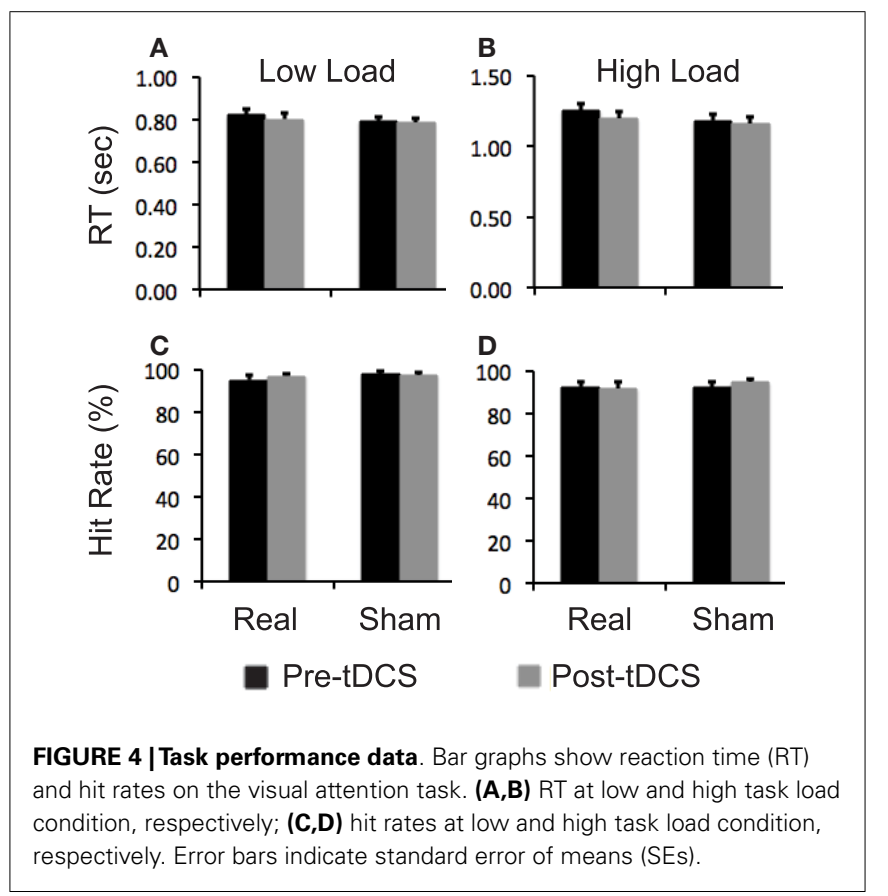

stimulation can reduce intracortical inhibition (74), and increase the functional activity of stimulated cortex (75-78). Several studies find that anodal stimulation of the left DLPFC reduces depressive symptoms of patients with major depression $(36,37,41$, 79), improves mood of patients with chronic tinnitus (80), and decreases negative ratings of pictures with negative valence in healthy participants (81). In the current study, real anodal stimulation of the left DLPFC significantly reduced negative affect of overnight abstinent smokers, and this effect positively correlated with the level of nicotine dependence, suggesting that tDCS is especially effective in heavy smokers. Based on above-reviewed literature, we predict that this effect of tDCS on negative affect is mediated by increased activity in the left DLPFC after anodal stimulation. This prediction can be tested in future studies using fMRI.

\section{CRAVING FOR SMOKING}

Different from no significant changes in negative affect after overnight abstinence, smokers reported a significant increase in craving for smoking. Real anodal stimulation of the left DLPFC did not significantly reduce craving relative to sham stimulation. This data is also different from the significant reduction in negative affect after real stimulation. Therefore, both the overnight abstinence and real anodal stimulation to the left DLPFC showed dissociable effects on negative affect vs. craving for smoking, indicating that the two common tobacco withdrawal symptoms have different neural mechanisms.

The current negative finding of tDCS on craving for smoking is different from previous findings of reduction in cue-induced craving after real tDCS $(22,23)$. A major difference between the current and the two previous tDCS studies is that the current study assessed overnight abstinent smokers while the previous studies assessed minimally abstinent $(\sim 1.5 \mathrm{~h})$ smokers. A recent study reported that overnight abstinence from smoking reduced the excitability increase of the primary motor cortex induced by anodal stimulation in tobacco dependent smokers, and that nicotine administration to overnight abstinent smokers reestablished the excitability increase of the motor cortex induced by anodal stimulation (82). Therefore, overnight abstinence from cigarette smoking may reduce the enhancing effect of anodal stimulation on cortical excitability in tobacco dependent smokers. This effect of overnight abstinence might contribute to the current negative finding on smoking craving after anodal stimulation of the left DLPFC. In addition, other factors, such as different participants, study procedures, and craving measures might also contribute to this different finding between current and previous studies.

Table 2 | Number of participants reported tDCS side effects.

\begin{tabular}{|c|c|c|c|c|c|c|c|c|c|}
\hline tDCS session & Headache & $\begin{array}{l}\text { Neck } \\
\text { pain }\end{array}$ & $\begin{array}{l}\text { Scalp } \\
\text { pain }\end{array}$ & $\begin{array}{l}\text { Scalp } \\
\text { burns }\end{array}$ & Tingling & $\begin{array}{l}\text { Skin } \\
\text { redness }\end{array}$ & Sleepiness & $\begin{array}{l}\text { Trouble } \\
\text { concentrating }\end{array}$ & $\begin{array}{l}\text { Acute mood } \\
\text { change }\end{array}$ \\
\hline Real (severity) & 0 & 0 & $1(2.0)$ & $4(2.3)$ & $19(2.1)$ & 0 & $7(2.6)$ & $1(2.0)$ & $1(3.0)$ \\
\hline Sham (severity) & $2(2.0)$ & $1(2.0)$ & $1(2.0)$ & $4(2)$ & $13(2.1)$ & $1(2.0)$ & $5(2.6)$ & $1(3.0)$ & $1(2.0)$ \\
\hline$X^{2}$ value & 2.18 & 1.07 & 0.001 & 0.004 & 2.77 & 1.07 & 0.341 & 0.001 & 0.001 \\
\hline$p$ & 0.140 & 0.302 & 0.975 & 0.947 & 0.096 & 0.301 & 0.559 & 0.975 & 0.975 \\
\hline
\end{tabular}

Number in the parenthesis indicates group mean of severity of reported side effect. 


\section{COGNITIVE FUNCTION}

No published studies have assessed the effect of tDCS on cognitive function of abstinent smokers. However, multiple tDCS studies report that anodal stimulation of the left DLPFC improves cognitive function including attention and working memory of healthy participants or patients with depression $(29,38,40,83,84)$. In the current study, anodal stimulation of the left DLPFC did not improve the performance of abstinent smokers on an attentional task. This negative finding may due to the specific task used in the current study, or other factors related to the negative finding on craving for smoking as discussed in the above section.

\section{tDCS SIDE EFFECT}

Consistent with previous tDCS studies, participants did not report serious side effects of tDCS in the current study. More participants reported tingling and sleepiness after real relative to sham stimulation. However, this difference between real and sham stimulation did not reach statistical significance.

\section{LIMITATIONS}

The main limitation of the current study is a small sample size and a short duration of abstinence. Tobacco withdrawal may

\section{REFERENCES}

1. Centers for Disease Control and Prevention (CDC). Annual smoking-attributable mortality, years of potential life lost, and economic costs - United States, 1995-1999. MMWR Morb Mortal Wkly Rep (2002) 51(4):300-3.

2. Centers for Disease Control and Prevention (CDC). Quitting smoking among adults - United States, 2001-2010. MMWR Morb Mortal Wkly Rep (2011) 60(44):1513-9.

3. DSM-IV. Diagnostic and Statistical Manual. 4th ed. Washington, DC: American Psychiatric Association (1994)

4. Henningfield JE, Shiffman S, Ferguson SG, Gritz ER. Tobacco dependence and withdrawal: science base, challenges and opportunities for pharmacotherapy. Pharmacol Ther (2009) 123:1-16. doi:10. 1016/j.pharmthera.2009.03.011

5. Xu J, Azizian A, Monterosso J, Domier CP, Brody AL, Fong TW, et al. Gender effects on mood and cigarette craving during early abstinence and resumption of smoking. Nicotine Tob Res (2008) 10:1653-61. doi:10.1080/ 14622200802412929

6. Cox LS, Nollen NL, Mayo MS, Choi WS, Faseru B, Benowitz NL, et al. Bupropion for smoking cessation in African American light smokers: a randomized controlled trial. $J$ Natl Cancer Inst (2012) 104:290-8. doi:10.1093/jnci/djr513

7. Heydari G, Talischi F, Tafti SF, Masjedi MR. Quitting smoking with varenicline: parallel, randomised efficacy trial in Iran. Int $J$ Tuberc Lung Dis (2012) 16:268-72. doi:10. 5588/ijtld.11.0183

8. Robles GI, Singh-Franco D, Ghin HL. A review of the efficacy of smoking-cessation pharmacotherapies in nonwhite populations. Clin Ther (2008) 30:800-12. doi:10. 1016/j.clinthera.2008.05.010

9. Shiffman S, Rolf CN, Hellebusch SJ, Gorsline J, Gorodetzky CW, Chiang YK, et al. Real-world efficacy of prescription and over-the-counter nicotine replacement therapy. Addiction (2002) 97:505-16. doi:10. 1046/j.1360-0443.2002.00141.x

10. Been G, Ngo TT, Miller SM, Fitzgerald PB. The use of tDCS and CVS as methods of non-invasive brain stimulation. Brain Res Rev (2007) 56:346-61. doi:10.1016/j. brainresrev.2007.08.001

11. Fregni F, Freedman S, PascualLeone A. Recent advances in the treatment of chronic pain with non-invasive brain stimulation techniques. Lancet $\mathrm{Neu}$ rol (2007) 6:188-91. doi:10.1016/ S1474-4422(07)70032-7

12. Nitsche MA, Cohen LG, Wassermann EM, Priori A, Lang N, Antal A, et al. Transcranial direct current stimulation: state of the art 2008 . Brain Stimul (2008) 1:206-23. doi: 10.1016/j.brs.2008.06.004

13. Stagg CJ, Nitsche MA. Physiological basis of transcranial direct current stimulation. Neuroscientist (2011) 17:37-53. doi:10.1177/ 1073858410386614

14. Utz KS, Dimova V, Oppenlander K, Kerkhoff G. Electrified

starts within a few hours of abstinence and will reach peak around $24 \mathrm{~h}$ after abstinence (68). Therefore, the effect of $\mathrm{tDCS}$ on negative affect should be assessed again when negative affect reaches its peak (e.g., after $24 \mathrm{~h}$ abstinence). Another limitation is no debriefing after the second tDCS, and therefore it is not clear whether the participants are really blind to the stimulation conditions.

In summary, tDCS is a safe, inexpensive, and easy to use method for modulating cortical excitability. Anodal stimulation of the left DLPFC showed a promising effect on negative affect of overnight abstinent smokers. Future studies should explore approaches, such as concurrent nicotine administration and behavioral therapy, or repeated sessions, for further enhancing the efficacy of anodal stimulation in ameliorating tobacco withdrawal in abstinent smokers.

\section{ACKNOWLEDGMENTS}

This study is supported by NIH grant K01DA027750. We thank Dr. Marc Potenza's support in study design, data acquisition, and manuscript preparation. We thank Dr. Stephanie O'Malley's for advice on study design issues. Clinical Trials.gov Identifier: NCT01567982.

minds: transcranial direct current stimulation (tDCS) and galvanic vestibular stimulation (GVS) as methods of non-invasive brain stimulation in neuropsychology - a review of current data and future implications. Neuropsychologia (2010) 48:2789-810. doi:10.1016/j. neuropsychologia.2010.06.002

15. Nitsche MA, Paulus W. Excitability changes induced in the human motor cortex by weak transcranial direct current stimulation. J Physiol (2000) 527(Pt 3):633-9. doi:10.1111/j.1469-7793.2000.t011-00633.x

16. Nitsche MA, Paulus W. Transcranial direct current stimulation update 2011. Restor Neurol Neurosci (2011) 29:463-92. doi:10. 3233/RNN-2011-0618

17. Monte-Silva K, Kuo MF, Liebetanz D, Paulus W, Nitsche MA. Shaping the optimal repetition interval for cathodal transcranial direct current stimulation (tDCS). J Neurophysiol (2010) 103:1735-40. doi:10. 1152/jn.00924.2009

18. Nitsche MA, Paulus W. Sustained excitability elevations induced by transcranial DC motor cortex stimulation in humans. Neurology (2001) 57:1899-901. doi:10.1212/WNL.57.10.1899

19. Ohn SH, Park CI, Yoo WK, Ko MH, Choi KP, Kim GM, et al. Time-dependent effect of transcranial direct current stimulation on the enhancement of working memory. Neuroreport (2008) 19:43-7. doi:10.1097/WNR. 0b013e3282f2adfd
20. Paulus W. Outlasting excitability shifts induced by direct current stimulation of the human brain. Suppl Clin Neurophysiol (2004) 57:708-14. doi:10.1016/S1567424X(09)70411-8

21. Alonzo A, Brassil J, Taylor JL, Martin D, Loo CK. Daily transcranial direct current stimulation (tDCS) leads to greater increases in cortical excitability than second daily transcranial direct current stimulation. Brain Stimul (2011) 5(3):208-13. doi:10.1016/j.brs. 2011.04.006

22. Boggio PS, Liguori P, Sultani N, Rezende L, Fecteau S, Fregni F. Cumulative priming effects of cortical stimulation on smoking cue-induced craving. Neurosci Lett (2009) 463:82-6. doi:10.1016/ j.neulet.2009.07.041

23. Fregni F, Liguori $P$, Fecteau $S$, Nitsche MA, Pascual-Leone A, Boggio PS. Cortical stimulation of the prefrontal cortex with transcranial direct current stimulation reduces cue-provoked smoking craving: a randomized, shamcontrolled study. J Clin Psychiatry (2008) 69:32-40. doi:10.4088/JCP. v69n0105

24. Boggio PS, Sultani N, Fecteau S, Merabet L, Mecca T, PascualLeone A, et al. Prefrontal cortex modulation using transcranial DC stimulation reduces alcohol craving: a double-blind, shamcontrolled study. Drug Alcohol Depend (2008) 92:55-60. doi:10.1016/j.drugalcdep.2007.06. 011 
25. Boggio PS, Zaghi S, Villani AB, Fecteau S, Pascual-Leone A, Fregni F. Modulation of risk-taking in marijuana users by transcranial direct current stimulation (tDCS) of the dorsolateral prefrontal cortex (DLPFC). Drug Alcohol Depend (2010) 112:220-5. doi:10.1016/j. drugalcdep.2010.06.019

26. Fregni F, Orsati F, Pedrosa W, Fecteau S, Tome FA, Nitsche MA, et al. Transcranial direct current stimulation of the prefrontal cortex modulates the desire for specific foods. Appetite (2008) 51:34-41. doi:10.1016/j.appet.2007. 09.016

27. Goldman RL, Borckardt JJ, Frohman HA, O’Neil PM, Madan A, Campbell LK, et al. Prefrontal cortex transcranial direct current stimulation (tDCS) temporarily reduces food cravings and increases the self-reported ability to resist food in adults with frequent food craving. Appetite (2011) 56:741-6. doi:10.1016/j.appet.2011.02.013

28. Montenegro RA, Okano AH, Cunha FA, Gurgel JL, Fontes EB, Farinatti PT. Prefrontal cortex transcranial direct current stimulation associated with aerobic exercise change aspects of appetite sensation in overweight adults. Appetite (2012) 58:333-8. doi:10.1016/j.appet.2011. 11.008

29. Andrews SC, Hoy KE, Enticott PG, Daskalakis ZJ, Fitzgerald PB. Improving working memory: the effect of combining cognitive activity and anodal transcranial direct current stimulation to the left dorsolateral prefrontal cortex. Brain Stimul (2011) 4:84-9. doi:10.1016/ j.brs.2010.06.004

30. Boggio PS, Bermpohl F, Vergara AO, Muniz AL, Nahas FH, Leme $\mathrm{PB}$, et al. Go-no-go task performance improvement after anodal transcranial DC stimulation of the left dorsolateral prefrontal cortex in major depression. J Affect Disord (2007) 101:91-8. doi:10.1016/j.jad. 2006.10.026

31. Bolognini N, Olgiati E, Rossetti A, Maravita A. Enhancing multisensory spatial orienting by brain polarization of the parietal cortex. Eur J Neurosci (2010) 31:1800-6. doi:10.1111/j. 1460-9568.2010.07211.x

32. Fregni F, Boggio PS, Nitsche $M$, Bermpohl F, Antal A, Feredoes E, et al. Anodal transcranial direct current stimulation of prefrontal cortex enhances working memory. Exp Brain Res (2005) 166:23-30. doi:10. 1007/s00221-005-2334-6
33. Jacobson L, Goren N, Lavidor M, Levy DA. Oppositional transcranial direct current stimulation (tDCS) of parietal substrates of attention during encoding modulates episodic memory. Brain Res (2012) 1439:66-72. doi:10.1016/j.brainres. 2011.12.036

34. Javadi AH, Walsh V. Transcranial direct current stimulation (tDCS) of the left dorsolateral prefrontal cortex modulates declarative memory. Brain Stimul (2011) 5(3):231-41. doi:10.1016/j.brs.2011.06.007

35. Leite J, Carvalho S, Fregni F, Goncalves OF. Task-specific effects of tDCS-induced cortical excitability changes on cognitive and motor sequence set shifting performance. PLoS One (2011) 6:e24140. doi:10. 1371/journal.pone.0024140

36. Loo CK, Alonzo A, Martin D, Mitchell PB, Galvez V, Sachdev P. Transcranial direct current stimulation for depression: 3-week, randomised, sham-controlled trial. $\mathrm{BrJ}$ Psychiatry (2012) 200:52-9. doi:10. 1192/bjp.bp.111.097634

37. Loo CK, Sachdev P, Martin D, Pigot M, Alonzo A, Malhi GS, et al. A double-blind, sham-controlled trial of transcranial direct current stimulation for the treatment of depression. Int J Neuropsychopharmacol (2010) 13:61-9. doi:10.1017/ S1461145709990411

38. Mulquiney PG, Hoy KE, Daskalakis ZJ, Fitzgerald PB. Improving working memory: exploring the effect of transcranial random noise stimulation and transcranial direct current stimulation on the dorsolateral prefrontal cortex. Clin Neurophysiol (2011) 122:2384-9. doi:10.1016/ j.clinph.2011.05.009

39. Nakamura-Palacios EM, de Almeida Benevides MC, da Penha ZagoGomes M, de Oliveira RW, de Vasconcellos VF, et al. Auditory eventrelated potentials (P3) and cognitive changes induced by frontal direct current stimulation in alcoholics according to Lesch alcoholism typology. Int $J$ Neuropsychopharmacol (2011) 15(5):601-16. doi:10.1017/S1461145711001040

40. Zaehle T, Sandmann P, Thorne JD, Jancke L, Herrmann CS. Transcranial direct current stimulation of the prefrontal cortex modulates working memory performance: combined behavioural and electrophysiological evidence. BMC Neurosci (2011) 12:2. doi:10.1186/14712202-12-2

41. Boggio PS, Rigonatti SP, Ribeiro RB, Myczkowski ML, Nitsche MA,
Pascual-Leone A, et al. A randomized, double-blind clinical trial on the efficacy of cortical direct current stimulation for the treatment of major depression. Int J Neuropsychopharmacol (2008) 11:249-54. doi:10.1017/S1461145707007833

42. Faber M, Vanneste S, Fregni F, De Ridder D. Top down prefrontal affective modulation of tinnitus with multiple sessions of tDCS of dorsolateral prefrontal cortex. Brain Stimul (2011) 5(4):492-8. doi:10. 1016/j.brs.2011.09.003

43. Kalu UG, Sexton CE, Loo CK, Ebmeier KP. Transcranial direct current stimulation in the treatment of major depression: a meta-analysis. Psychol Med (2012) 42(9):1791-800. doi:10.1017/S0033291711003059

44. Martin DM, Alonzo A, Mitchell PB, Sachdev P, Galvez V, Loo CK. Fronto-extracephalic transcranial direct current stimulation as a treatment for major depression: an open-label pilot study. J Affect Disord (2011) 134:459-63. doi:10. 1016/j.jad.2011.05.018

45. Nitsche MA, Boggio PS, Fregni F, Pascual-Leone A. Treatment of depression with transcranial direct current stimulation (tDCS): a review. Exp Neurol (2009) 219:14-9. doi:10.1016/j.expneurol. 2009.03.038

46. Heatherton TF, Kozlowski LT, Frecker RC, Fagerström KO. The Fagerström test for nicotine dependence: a revision of the Fagerström tolerance questionnaire. Br J Addict (1991) 86:1119-27.

47. Jarvik M, Madsen D, Olmstead $\mathrm{R}$, Iwamoto-Schaap P, Elins J, Benowitz NL. Nicotine blood levels and subjective craving for cigarettes. Pharmacol Biochem Behav (2000) 66:553. doi:10.1016/S00913057(00)00261-6

48. McNair D, Lorr M, Droppleman L. Profile of Mood States (Manual). San Diego: Educational and Industrial Testing Service (1971).

49. Fregni F, Boggio PS, Lima MC, Ferreira MJ, Wagner T, Rigonatti SP, et al. A sham-controlled, phase II trial of transcranial direct current stimulation for the treatment of central pain in traumatic spinal cord injury. Pain (2006) 122:197-209. doi:10.1016/j.pain. 2006.02.023

50. Boggio PS, Ferrucci R, Rigonatti SP, Covre P, Nitsche M, PascualLeone A, et al. Effects of transcranial direct current stimulation on working memory in patients with Parkinson's disease. J Neurol Sci
(2006) 249:31-8. doi:10.1016/j.jns. 2006.05.062

51. Brunoni AR, Amadera J, Berbel B, Volz MS, Rizzerio BG, Fregni F. A systematic review on reporting and assessment of adverse effects associated with transcranial direct current stimulation. Int $J$ Neuropsychopharmacol (2011) 14:1133-45. doi:10.1017/S1461145710001690

52. Gandiga PC, Hummel FC, Cohen LG. Transcranial DC stimulation (tDCS): a tool for double-blind sham-controlled clinical studies in brain stimulation. Clin Neurophysiol (2006) 117:845-50. doi:10.1016/ j.clinph.2005.12.003

53. Lavie N. The role of perceptual load in visual awareness. Brain Res (2006) 1080:91-100. doi:10.1016/j. brainres.2005.10.023

54. Xu J, Monterosso J, Kober H, Balodis IM, Potenza MN. Perceptual loaddependent neural correlates of distractor interference inhibition. PLoS One (2011) 6:e14552. doi:10.1371/ journal.pone.0014552

55. Preacher K. Calculation for the Test of the Difference Between Two Independent Correlation Coefficients [Computer software]. (2002). Available from: http:/quantpsy.org

56. Brody AL, Mukhin AG, Shulenberger S, Mamoun MS, Kozman M, Phuong J, et al. Treatment for tobacco dependence: effect on brain nicotinic acetylcholine receptor density. Neuropsychopharmacology (2013) 38(8):1548-56. doi:10. 1038/npp. 2013.53

57. Gentry CL, Lukas RJ. Regulation of nicotinic acetylcholine receptor numbers and function by chronic nicotine exposure. Curr Drug Targets CNS Neurol Disord (2002) 1:359-85. doi:10.2174/ 1568007023339184

58. Groman E, Fagerstrom K. Nicotine dependence: development, mechanisms, individual differences and links to possible neurophysiological correlates. Wien Klin Wochenschr (2003) 115:155-60. doi:10. 1007/BF03040301

59. Sabbagh MN, Lukas RJ, Sparks DL, Reid RT. The nicotinic acetylcholine receptor, smoking, and Alzheimer's disease. J Alzheimers Dis (2002) 4:317-25.

60. Brody AL, Mandelkern MA, Jarvik ME, Lee GS, Smith EC, Huang JC, et al. Differences between smokers and nonsmokers in regional gray matter volumes and densities. Biol Psychiatry (2004) 55:77. doi:10.1016/S0006-3223(03) 00610-3 
61. Gallinat J, Meisenzahl E, Jacobsen LK, Kalus P, Bierbrauer J, Kienast $\mathrm{T}$, et al. Smoking and structural brain deficits: a volumetric MR investigation. Eur J Neurosci (2006) 24:1744-50. doi:10.1111/j. 1460-9568.2006.05050.x

62. Sutherland MT, Ross TJ, Shakleya DM, Huestis MA, Stein EA. Chronic smoking, but not acute nicotine administration, modulates neural correlates of working memory. Psychopharmacology (Berl) (2011) 213:29-42. doi:10. 1007/s00213-010-2013-6

63. Beaver JD, Long CJ, Cole DM, Durcan MJ, Bannon LC, Mishra RG, et al. The effects of nicotine replacement on cognitive brain activity during smoking withdrawal studied with simultaneous fMRI/EEG. Neuropsychopharmacology (2011) 36:1792-800. doi:10. 1038/npp.2011.53

64. Cole DM, Beckmann CF, Long CJ, Matthews PM, Durcan MJ, Beaver JD. Nicotine replacement in abstinent smokers improves cognitive withdrawal symptoms with modulation of resting brain network dynamics. Neuroimage (2010) 52:590-9. doi:10.1016/j. neuroimage.2010.04.251

65. Goldstein RZ, Volkow ND. Dysfunction of the prefrontal cortex in addiction: neuroimaging findings and clinical implications. Nat Rev Neurosci (2011) 12:652-69. doi:10. 1038/nrn3119

66. Xu J, Mendrek A, Cohen MS, Monterosso J, Rodriguez P, Simon SL, et al. Brain activity in cigarette smokers performing a working memory task: effect of smoking abstinence. Biol Psychiatry (2005) 58:143. doi: 10.1016/j.biopsych.2005.03.028

67. Xu J, Mendrek A, Cohen MS, Monterosso J, Simon S, Brody AL, et al. Effects of acute smoking on brain activity vary with abstinence in smokers performing the N-back task: a preliminary study. Psychiatry
Res (2006) 148:103-9. doi:10.1016/ j.pscychresns.2006.09.005

68. Hughes JR. Effects of abstinence from tobacco: valid symptoms and time course. Nicotine Tob Res (2007) 9:315-27. doi:10.1080/ 14622200701188919

69. West R, Hajek P. Evaluation of the mood and physical symptoms scale (MPSS) to assess cigarette withdrawal. Psychopharmacology (Berl) (2004) 177:195-9. doi:10. 1007/s00213-004-1923-6

70. Habel U, Klein M, Kellermann T, Shah NJ, Schneider F. Same or different? Neural correlates of happy and sad mood in healthy males. Neuroimage (2005) 26:206-14. neuroimage.2005.01.014

71. Herrington JD, Mohanty A, Koven NS, Fisher JE, Stewart JL, Banich MT, et al. Emotion-modulated performance and activity in left dorsolateral prefrontal cortex. Emotion (2005) 5:200-7. doi:10.1037/15283542.5.2.200

72. Sergerie K, Lepage M, Armony JL. A face to remember: emotional expression modulates prefrontal activity during memory formation. Neuroimage (2005) 24:580-5. doi: 10.1016/j.neuroimage.2004.08.051

73. Schutter DJ, van Honk J. A framework for targeting alternative brain regions with repetitive transcranial magnetic stimulation in the treatment of depression. J Psychiatry Neurosci (2005) 30:91-7.

74. Antal A, Terney D, Kuhnl S, Paulus W. Anodal transcranial direct current stimulation of the motor cortex ameliorates chronic pain and reduces short intracortical inhibition. I Pain Symptom Manage (2010) 39:890-903. doi:10.1016/j. jpainsymman.2009.09.023

75. Keeser D, Meindl T, Bor J, Palm U, Pogarell O, Mulert C, et al. Prefrontal transcranial direct current stimulation changes connectivity of resting-state networks during fMRI.
J Neurosci (2011) 31:15284-93. doi:10.1523/JNEUROSCI.0542-11. 2011

76. Meinzer M, Antonenko D, Lindenberg R, Hetzer S, Ulm L, Avirame $\mathrm{K}$, et al. Electrical brain stimulation improves cognitive performance by modulating functional connectivity and task-specific activation. J Neurosci (2012) 32:1859-66. doi:10. 1523/JNEUROSCI.4812-11.2012

77. Merzagora AC, Foffani G, Panyavin I, Mordillo-Mateos L, Aguilar J, Onaral B, et al. Prefrontal hemodynamic changes produced by anodal direct current stimulation. $\mathrm{Neu}$ roimage (2010) 49:2304-10. doi:10. 1016/j.neuroimage.2009.10.044

78. Polania R, Nitsche MA, Paulus W. Modulating functional connectivity patterns and topological functional organization of the human brain with transcranial direct current stimulation. Hum Brain Mapp (2011) 32:1236-49. doi:10.1002/ hbm. 21104

79. Ferrucci R, Bortolomasi M, Vergari M, Tadini L, Salvoro B, Giacopuzzi $\mathrm{M}$, et al. Transcranial direct current stimulation in severe, drugresistant major depression. J Affect Disord (2009) 118:215-9. doi:10. 1016/j.jad.2009.02.015

80. Frank E, Schecklmann M, Landgrebe M, Burger J, Kreuzer P, Poeppl $\mathrm{TB}$, et al. Treatment of chronic tinnitus with repeated sessions of prefrontal transcranial direct current stimulation: outcomes from an open-label pilot study. $\mathrm{J} \mathrm{Neu}$ rol (2012) 259:327-33. doi:10.1007/ s00415-011-6189-4

81. Pena-Gomez C, Vidal-Pineiro D, Clemente IC, Pascual-Leone A Bartres-Faz D. Down-regulation of negative emotional processing by transcranial direct current stimulation: effects of personality characteristics. PLoS One (2011) 6:e22812. doi:10.1371/journal.pone.0022812

82. Grundey J, Thirugnanasambandam N, Kaminsky K, Drees A, Skwirba
AC, Lang N, et al. Neuroplasticity in cigarette smokers is altered under withdrawal and partially restituted by nicotine exposition. J Neurosci (2012) 32:4156-62. doi:10.1523/ JNEUROSCI.3660-11.2012

83. Kuo MF, Nitsche MA. Effects of transcranial electrical stimulation on cognition. Clin EEG Neurosci (2012) 43:192-9. doi:10.1177/ 1550059412444975

84. Wolkenstein L, Plewnia C. Amelioration of cognitive control in depression by transcranial direct current stimulation. Biol Psychiatry (2013) 73:646-51. doi:10.1016/ j.biopsych.2012.10.010

Conflict of Interest Statement: The authors declare that the research was conducted in the absence of any commercial or financial relationships that could be construed as a potential conflict of interest.

Received: 13 June 2013; accepted: 04 September 2013; published online: 20 September 2013.

Citation: $X u$ J, Fregni F, Brody $A L$ and Rahman AS (2013) Transcranial direct current stimulation reduces negative affect but not cigarette craving in overnight abstinent smokers. Front. Psychiatry 4:112. doi: 10.3389/fpsyt.2013.00112

This article was submitted to Addictive Disorders and Behavioral Dyscontrol, a section of the journal Frontiers in Psychiatry.

Copyright $\odot 2013$ Xu, Fregni, Brody and Rahman. This is an open-access article distributed under the terms of the Creative Commons Attribution License (CC $B Y)$. The use, distribution or reproduction in other forums is permitted, provided the original author(s) or licensor are credited and that the original publication in this journal is cited, in accordance with accepted academic practice. No use, distribution or reproduction is permitted which does not comply with these terms. 